\title{
Is GABA Co-Released with Glutamate from Hippocampal Mossy Fiber Terminals?
}

\author{
Maddalena Delma Caiati \\ International School for Advanced Studies (SISSA), Neuroscience Area, Trieste 34136, Italy \\ Review of Cabezas et al.
}

The coexistence and co-release of different neurotransmitters from the same fiber has been well documented in several brain structures, including the retina, the spinal cord, and the auditory system (Hnasko and Edwards, 2012). Although mossy fibers (MFs), the axons of dentate gyrus granule cells, release glutamate and GABA early in postnatal development (Walker et al., 2001; Gutiérrez et al., 2003; Safiulina et al., 2006), they normally provide monosynaptic glutamatergic excitation and disynaptic GABAergic inhibition to the CA3 hippocampal field in adults. But in some conditions, such as kindling or activitydependent processes, MFs can transiently resume a GABAergic phenotype in adulthood. This suggests that MFs can switch, in a developmentally and activity-dependent regulated way, the type of neurotransmitter released (Gutiérrez, 2005).

Studies using immunohistochemistry and electron microscopy have revealed that MFs possess the full machinery to synthesize, store, and release GABA. Glutamic acid decarboxylase (GAD67/65), the enzyme that catalyzes GABA synthesis, as well as GABA itself and the vesicular GABA transporter, VGAT, have all been detected within MF terminals. Importantly, immunogold experiments have demon-

Received Oct. 26, 2012; revised Nov. 29, 2012; accepted Nov. 30, 2012.

I acknowledge Prof. Enrico Cherubini for his helpful review of the manuscript.

Correspondence should be addressed to Maddalena Delma Caiati, SISSA, Via Bonomea 265, Trieste (TS) 34136, Italy. E-mail: caiati@sissa.it. DOI:10.1523/JNEUROSCI.5019-12.2013

Copyright $\odot 2013$ the authors $\quad 0270-6474 / 13 / 331755-02 \$ 15.00 / 0$ strated that AMPA and $\mathrm{GABA}_{\mathrm{A}}$ receptors are sometimes colocalized on postsynaptic sites in close apposition to MF terminals, strongly suggesting that MFs may convey a GABAergic signal to their targets (Gutierrez, 2005). Consistent with this hypothesis, monosynaptic GABAergic currents have been recorded in CA3 principal cells upon granule cell stimulation in the dentate gyrus in acute hippocampal slices from newborn (Safiulina et al., 2006) or juvenile animals (Walker et al., 2001; Gutiérrez et al., 2003). The evoked responses fulfill the criteria for identification of MF inputs: strong paired pulse facilitation, short term frequencydependent facilitation, and sensitivity to group II and III mGluR agonists (Safiulina et al., 2006).

The data discussed above have been challenged, however. Specifically, Uchigashima et al. (2007) questioned the GABAergic nature of MF-CA3 responses on the basis that, at least in young animals, the stimulation protocol generally used to activate MFs might coactivate adjacent GABAergic terminals, thus causing misinterpretation of the results. Given the complex nature of the neuronal network of the dentate gyrus and CA3 area, the possibility that interneurons with axonal or dendritic projections to the dentate gyrus could be activated with minimal stimulation protocol is plausible. This controversial issue may be solved by performing paired recordings from interconnected granule cells and CA3 principal neurons.

In a study recently published in The Journal of Neuroscience, Cabezas et al. (2012) used paired recordings of interconnected neurons in organotypic hippocampal slice cultures from GAD67-EGFP transgenic mice to explore the possibility that MFs can co-release glutamate and GABA onto CA3 principal cells. First, by performing immunocytochemical experiments from the hippocampus of postnatal day 15 (P15) old mice, the authors found that GAD67 is expressed only in a subset of MF terminals immunopositive for $\mathrm{ZnT3}$, a selective $\mathrm{MF}$ marker. Interestingly, GAD67-positive granule cells showed signs of immaturity, including expression of doublecortin but not calbindin, low membrane capacitance, moderate input resistance, and small amplitude action potentials.

Next, in organotypic hippocampal slices prepared from P7-P8 old mice and kept in cultures for 10-12 days, Cabezas et al. (2012) used local photolysis of caged glutamate to examine postsynaptic responses evoked by photostimulation of individual, visually identified granule cells. Despite the expression of GAD67 in a subpopulation of immature granule cells, no unitary $\mathrm{GABA}_{\mathrm{A}}$-mediated synaptic currents were detected in any of the 39 pairs of interconnected granule cells and CA3 principal cells examined, casting doubts on previous findings obtained from acute hippocampal slices (Walker et al., 2001; Gutiérrez et al., 2003; Safiulina et al., 2006). Based on their reversal potential and pharmacology, unitary currents were identified as mediated by AMPA/ kainate receptors. 
Notwithstanding the lack of GABAmediated postsynaptic currents, however, Cabezas et al. (2012) showed that GAD67positive granule cells release GABA. They demonstrated that GABA released from MF terminals acts upon presynaptic $\mathrm{GABA}_{\mathrm{B}}$ receptors, reducing MF excitability. Thus, repetitive activation of EGFPpositive neurons in the dentate gyrus (a train of 30 orthodromic action currents at $25 \mathrm{~Hz}$ elicited by depolarizing voltage pulses through the patch pipette) transiently reduced the probability of evoking antidromic action currents by extracellular stimulation of MFs in stratum lucidum, and this effect was blocked by selective $\mathrm{GABA}_{\mathrm{B}}$ receptor antagonists. Therefore, it appears that, in juvenile animals, a transient MF GABAergic phenotype is important for instructing presynaptic rather than postsynaptic elements of synapses.

Although the data presented by Cabezas et al. (2012) favor the hypothesis that monosynaptic GABAergic currents recorded from CA3 pyramidal cells in acute slices (upon stimulation of granule cells) originate from direct activation of hilar interneurons and not dentate gyrus granule cells, it is worth noting that Cabezas et al. (2012) used a different preparation than previous studies. Specifically, whereas earlier studies (Walker et al., 2001; Gutiérrez et al., 2003; Safiulina et al., 2006) used acute hippocampal slices, Cabezas et al. (2012) used organotypic hippocampal slices kept in cultures for 10-12 days before recording. Because it is impossible to determine the exact developmental stage of MFs in culture, making a direct comparison between the two preparations is extremely difficult. Furthermore, although organotypic hippocampal slices maintain some local circuitry intact, they develop in isolation and hence they lack the experience-dependent plasticity characteristic of behaving animals. Because MF-dependent GABAergic transmission is strongly activity dependent (Gutierrez et al., 2003), the lack of extrinsic afferents in slice cultures may interfere with the acquisition of a functional GABAergic phenotype from granule cells. In addition, compared to acute slices, organotypic cultures exhibit enhanced glutamatergic connectivity as demonstrated by the four- to five-fold increase in the frequency of gluta- matergic but not GABAergic miniature postsynaptic currents (De Simoni et al., 2003). Moreover, the fact that astrocytes do not reach a full maturation in organotypic cultures (Derouiche, 1993) might alter the normal glutamate and GABA metabolism, possibly lowering the level of GABA released from MF terminals. Finally, it is unclear whether, $\mathrm{GABA}_{\mathrm{A}}$ receptors facing MF terminals remain present and functional in organotypic cultures.

In addition to the caveats listed above, the observations of Cabezas et al. (2012) are difficult to reconcile with a recent report by Beltran and Gutiérrez (2012), which examined synaptic responses evoked by stimulation of single identified MF boutons attached to the apical dendrites of mechanically isolated pyramidal cells. Such stimulation produced synaptic currents that, like typical MF-evoked responses, showed a high degree of facilitation upon repetitive stimulation and were blocked by group II mGluR agonists. Interestingly, whereas stimulation of MF boutons in neurons dissociated from adult animals generated exclusively glutamate receptor-mediated responses, stimulation of MF boutons from younger animals produced either GABAergic or mixed GABAergic and glutamatergic responses.

In summary, the question of whether activation of immature MF terminals elicits monosynaptic GABAergic responses in CA3 pyramidal cells remains unsolved. Recently developed tools may help to clarify this issue. For example, expressing channelrhodopsin-2 (ChR2) via a retroviral vector in hippocampal granule cell progenitors would allow one to selectively activate ChR2-positive granule cells by photostimulation and to identify the nature of the neurotransmitter released by MFs onto patched CA3 principal cells. Classical criteria for MF identification could then be used to ascertain that ChR2driven synaptic responses were truly MFs.

Alternatively, by sequentially uncaging glutamate with the beam-multiplexed two-photon laser, a novel optical method developed by Nikolenko et al. (2007), it would be possible to selectively activate (in acute hippocampal slices from GAD67EGFP transgenic mice) up to a thousand
GAD67-positive granule cells, causing them to fire. This would allow investigation of the nature of monosynaptic-evoked responses in CA3-targeted pyramidal cells.

Either of these approaches would permit one to selectively activate granule cells, as well as to overcome the difficulty of finding connected pairs of neurons. Such experiments might finally elucidate the role of GABA in MF terminals.

\section{References}

Beltrán JQ, Gutiérrez R (2012) Co-release of glutamate and GABA from single, identified mossy fibre giant boutons. J Physiol 590: 4789-4800. CrossRef Medline

Cabezas C, Irinopoulou T, Gauvain G, Poncer JC (2012) Presynaptic but not postsynaptic GABA signaling at unitary mossy fiber synapses. J Neurosci 32:11835-11840. CrossRef Medline

Derouiche A, Heimrich B, Frotscher M (1993) Loss of layer-specific astrocytic glutamine synthetase immunoreactivity in slice cultures of hippocampus. Eur J Neurosci 5:122-127. CrossRef Medline

De Simoni A, Griesinger CB, Edwards FA (2003) Development of rat CA1 neurons in acute versus organotypic slices: role of experience in synaptic morphology and activity. J Physiol 550:135-147. CrossRef Medline

Gutiérrez R (2005) The dual glutamatergicGABAergic phenotype of hippocampal granule cells. Trends Neurosci 28:297-303. CrossRef Medline

Gutiérrez R, Romo-Parra H, Maqueda J, Vivar C, Ramìrez M, Morales MA, Lamas M (2003) Plasticity of the GABAergic phenotype of the "glutamatergic" granule cells of the rat dentate gyrus. J Neurosci 23:5594-5598. Medline

Hnasko TS, Edwards RH (2012) Neurotransmitter co-release: mechanism and physiological role. Annu Rev Physiol 74:225-243. CrossRef Medline

Nikolenko V, Poskanzer KE, Yuste R (2007) Two-photon photostimulation and imaging of neural circuits. Nat Methods 4:943-950. CrossRef Medline

Safiulina VF, Fattorini G, Conti F, Cherubini E (2006) GABAergic signaling at mossy fiber synapses in neonatal rat hippocampus. J Neurosci 26:597-608. CrossRef Medline

Uchigashima M, Fukaya M, Watanabe M, Kamiya H (2007) Evidence against GABA release from glutamatergic mossy fiber terminals in the developing hippocampus. J Neurosci 27: 8088-8100. CrossRef Medline

Walker MC, Ruiz A, Kullmann DM (2001) Monosynaptic GABAergic signaling from dentate to CA3 with a pharmacological and physiological profile typical of mossy fiber synapses. Neuron 29:703-715. CrossRef Medline 